\title{
CONSEILLERS SCÉLÉRATS ET MAUVAIS GOUVERNEMENT : LE ROI D'ARAGON, SES CONSEILLERS ET LE CONSEIL À LA FIN DU XIVe SIÈCLE
}

\author{
Alexandra Beauchamp \\ Université de Limoges / EA 4270 \\ Faculté des Lettres et des Sciences Humaines
}

\section{RESUMEN}

Este artículo constituye un preámbulo al análisis del gobierno a través del Consejo por parte de los monarcas de la Corona de Aragón a fines del siglo XIV. En las Cortes Generales de Monzón de 1383 los súbditos protestaron contra la posición y las decisiones de los consejeros reales y pusieron en cuestionamiento la manera en que el Consejo ejercía su poder durante el reinado del rey Ceremonioso. De hecho, diferían completamente de las teorizaciones realizadas por el rey sobre el comportamiento de los consejeros. No en vano, las ordenanzas reales (las Ordinacions de la Casa i Cort de 1344) enfatizaban que debían tener unas competencias reducidas y que sus discursos y acciones debían ser cuidadosamente controlados.

Palabras clave: Consejeros; Consejo real; Ordenanzas; Cortes; Corona de Aragón.

\section{ABSTRACT}

This paper is a preamble to a study of the " gouvernement par conseil » of the king of Aragon at the end of the XIV ${ }^{\text {th }}$ Century. In 1383, in Monzón, during the Cortes generales, the subjects protested against the position 
and decisions of the royal councillors; they challenged the way Peter the Ceremonious applied this kind of government. De facto, it differed totally from the way the king had theorized about his councillors' intervention. An analysis of the royal ordinances (Ordinacions de la Casa i Cort, 1344) emphasizes that they should have a reduced scope and that their speech and action should be carefully controlled.

Keywords: Councillors; Royal Council; Ordinances; Parliament; Crown of Aragon.

\section{RÉSUMÉ}

Cet article constitue le préambule d'une analyse du gouvernement par conseil, tel que le roi d'Aragon l'applique à la fin du XIV siècle. Il montre combien lors des Cortes generales de Monzón, en 1383, les sujets contestent la place et les décisions des conseillers royaux et remettent en cause la façon dont le gouvernement par conseil s'exerçait sous le règne de Pierre le Cérémonieux. De fait, cet exercice du pouvoir diffère radicalement de la façon dont le roi avait théorisé l'intervention de ses conseillers. L'examen des ordonnances royales (Ordinacions de la Casa i Cort, 1344) souligne en effet la latitude normalement assez réduite qui devait être la leur et la façon dont leur parole et leur action devaient soigneusement être encadrées.

Mots clés: Conseillers ; Gouvernement par conseil ; Ordonnances ; Cortes generales; Couronne d'Aragon.

Cet article constitue le préambule d'une analyse du gouvernement par conseil, tel que le roi d'Aragon l'applique à la fin du XIV siècle $^{1}$. Avant de

1 Dans la Couronne d'Aragon, le conseil royal a fait l'objet de rares études, qui n'envisagent qu'à la marge la question du gouvernement par conseil : SEVILLANO COLOM, F., « Apuntes para el estudio de la cancillería de Pedro IV el Cermonioso », Anales de historia del derecho español, 20 (1950), pp. 137-241 donne quelques éléments sur le rôle du personnel de la chancellerie au conseil ; TATJER PRAT, M. T., « La potestad judicial del rey. El consejo del rey en su función de administrar justicia (s. XIII y XIV) », dans El poder real en la Corona de Aragón (s. XIV-XVI). Actas del XV Congreso de Historia de la Corona de Aragón (Jaca 1993), Saragosse, 1996, t. 1, vol. 2, pp. 378-388, étudie le rôle de tribunal suprême de cet organe ; FERRER I MALLOL, M. T., «El consell reial durant el regnat de Martí l'humà », dans El poder real en la Corona de Aragón (s. XIV-XVI). Actas del XV $\mathrm{XV}^{\circ}$ Congreso de Historia de la Corona de Aragón (Jaca 1993), Saragosse, 1996, t.1, vol. 2, pp. 175-190 et CORRAO, P., Governare un regno. Potere, società e istituzioni in Sicilia fra Trecento e Quattrocento, Naples, 1991, pp. 261306, s'intéressent à sa composition ; le second montre le rôle majeur de cet organe dans le gouvernement du royaume aragonais de Sicile, entre la fin du XIV siècle et le premier quart du $\mathrm{XV}^{\mathrm{e}}$ siècle et explique comment sa composition et le recrutement de 
traiter, dans une prochaine étude, de l'importance effective des conseillers, de leur recrutement et du fonctionnement pratique du conseil royal, cet « organe essentiel de la vie de la monarchie », j'ai choisi, dans les pages qui suivent, d'étudier les discours formulés sur le rôle des conseillers ${ }^{2}$. Partant de l'exemple des Cortes generales de Monzón, en 1383, je souligne combien les sujets y contestent la place et les décisions des conseillers royaux ; l'influence de ces derniers étant jugée nocive sur la politique du souverain, les sujets souhaitent la limiter. De fait, l'exercice du pouvoir qu'ils décrivent diffère radicalement de la façon dont le roi avait théorisé l'intervention de ses conseillers. L'examen des ordonnances royales (Ordinacions de la Casa i Cort, 1344) montre en effet la latitude normalement assez réduite qui devait être la leur et la façon dont leur parole et leur action devaient soigneusement être encadrées.

\section{DE MAUVAIS CONSEILS PRODIGUÉS PAR DE MAUVAIS CONSEILLERS : LES DÉNONCIATIONS DES CORTES GENERALES DE MONZÓN}

Le 12 juin 1383, à Monzón en Aragon, le roi Pierre le Cérémonieux ouvrait solennellement les travaux des Cortes generales de tous ses royaumes et territoires, par un élégant discours (proposición) ${ }^{3}$. En roi soucieux de ses

conseillers issus de l'aristocratie sicilienne participent de la politique d'intégration du royaume à la Couronne d'Aragon ; dans cette même lignée, voir OLIVA, A. M., « Il consiglio regio nel regno di Sardegna. Prime ricerche ", dans Ferrer i Mallol, M. T., Mutgé i Vives, J., Sánchez Martínez, M., La Corona catalanoaragonesa i el seu entorn mediterrani a la Baixa Edat Mitjana, Barcelone, 2005, pp. 205-238 ; ABADAL, R. d', Pere el Cerimoniós i els inicis de la decadència política de Catalunya, Barcelone, 1987, pp. 80-88 analyse la place du conseil royal et sa composition dans la chronique de Pierre IV; le rôle de son conseiller Bernat de Cabrera a été récemment étudié par BÉHROUZI, M., Le procès fait à Bernat de Cabrera (1364-1372), thèse inédite soutenue le 15 février 2014, à l'université de Bordeaux III sous la direction de F. Lainé. Sur le gouvernement par conseil en général et le cas français en particulier, voir CANTEAUT, O., « Le roi de France gouverne-t-il par conseil ? L'exemple de Philippe V », dans Charageat, M., Leveleux-Texeira, C., Consulter, délibérer, décider : donner son avis au Moyen Âge, Toulouse, 2010, pp. 157-176.

2 Lexpression est de ABADAL, R. d', Pere el Cerimoniós, p. 81.

3 Le proceso de cette assemblée est édité par SANS I TRAVÉ, J. M., Cort General de Montsó, 1382-1384, Barcelone, 1992, ainsi que par SESMA MUÑOZ, J. Á. (éd.), Acta curiarum Aragonum, t. V : Cortes de los reinados de Pedro IV/4 y Juan I. Cortes de Zaragoza 1381, Cortes generales de Monzón, Tamarite de Litera y Fraga 1383-1384, Cortes generales de Monzón, 1388-1389, Saragosse, 2009, pp. 153-275. Le discours du roi est reproduit respectivement pp. 78-81 et pp. 192-193, ainsi que dans ALBERT, R., GASSIOT, J., Parlaments a les Corts catalanes, Barcelone, 1928, pp. 52-65. Sur l'implication de Pierre IV dans la rédaction de ses discours, voir GIMENO BLAY, F., Escribir, 
sujets aragonais, valenciens, catalans et majorquins qui avaient demandé à être réunis, il s'y disait disposé à écouter leurs requêtes et à agir au mieux pour le service de Dieu, le profit et l'intérêt de sa terre. La réponse de l'assemblée, véritable plaidoyer pour la justice, intervint le 27 juin lorsqu'une cédule écrite, présentée par l'infant Martin au nom des Cortes, fut lue devant le souverain ${ }^{4}$. Les représentants des sujets y dénonçaient les abus et injustices commis par « molts mals homens de consell e de casa del dit senyor duch e encara de consell e casa de vos, senyor $»$. Selon les termes de cette cédule, des proches de Pierre IV et de son fils aîné et successeur désigné, l'infant Jean, duc de Gérone, se seraient unis et auraient fomenté une cabale sanctionnée par un serment aux dépens des intérêts du roi et de ses sujets ${ }^{5}$. Lassemblée affirmait l'influence générale néfaste sur le gouvernement, en termes diplomatique et militaire, matériel, politique et judiciaire, de ces mauvais conseillers - membres ou non des Hôtels royal et ducal-; elle invitait le roi à les exclure de son conseil, de sa Maison et de ceux du duc et à les châtier pour que « la vostra reyal cort sia purgada de tals persones scelerades ». Dans un contexte politique et militaire délicat, les sujets accusaient en effet ces hommes de trahison en affirmant qu'ils informaient outrageusement l'ennemi, lui ouvraient les portes des territoires de la Couronne d'Aragon ${ }^{6}$; ils les taxaient en outre

reinar. La experiencia gráfico-textual de Pedro IV, Madrid, 2006, pp. 137-145 ; sur l'art oratoire des souverains aragonais, voir CAWSEY, S. F., Kingship and Propaganda. Royal Eloquence and the Crown of Aragon c.1200-1450, Oxford, 2002 et en dernier lieu, le nouvel ouvrage de HÉBERT, M., Parlementer. Assemblées représentatives et échange politique en Europe occidentale à la fin du Moyen Âge, Paris, 2014, pp. 344-347 ; il consacre des pages lumineuses (pp. 55-77) à ces Cortes generales, qui avant lui n'avait guère été étudiées, outre l'article de SESMA MUÑOZ, J. Á. , " Todos frente al rey. La oposición al establecimiento de una monarquía centralizada en la Corona de Aragón a finales del siglo XIV », dans Rucquoi, A. (coord.), Genèse médiévale de l'Espagne moderne. Du refus à la révolte: les resistances, Nice, 1991, pp. 86-87.

4 Reproduite dans SANS I TRAVÉ, Cort General de Montsó, pp. 100-104 et J. M., SESMA MUÑOZ, J. Á. (éd.), Acta curiarum Aragonum, pp. 203-205, édition citée ci-après.

5 « alcuns del consell del dit senyor duch e del vostre consell, per mils acabar les dites e altres malvestats e avoleses se son units e agabebellats, estrenyents se entre si sots virtut de sagrament e homenatge de esser tots una cosa e dun cor, e voler no noure en res uns a altres, e faents entre si altres empreniments e permissions no legudes en cascun consell del dit senyor duch e vostre $»$.

$6 \quad$ Ils seraient coupables de " descobrir e revelar los secrets e consells e affers de vos senyor ab letres e en altres maneres a vostres enemichs e malvolents ", de " fer metre dins vostra senyoria per via d'armes e de guerra vostres enemichs ». Sur ce contexte : BOSCOLO, A., « Problemi mediterranei dell'epoca di Pietro il Ceremonioso (13531387) », dans La Corona de Aragón en el siglo XIV. Actas del VIII ${ }^{\circ}$ Congreso de historia de la Corona de Aragón, (Valencia 1967), Valence, 1973, t. II, vol. 3, pp. 65-99 et MELONI, G., Genova e Aragona all'epoca di Pietro il Ceremonioso, vol. 3 : 1361-1387, Padoue, 1971. 
d'être de fort mauvais conseil car ils auraient abusivement poussé à des négociations de paix coûteuses et peu nécessaires avec les ennemis génois ${ }^{7}$. Tout aussi grave était la responsabilité que les Cortes leurs attribuait dans l'affaiblissement périlleux des moyens matériels du souverain ${ }^{8}$, puisque selon eux, pour s'enrichir personnellement, ils n'auraient pas craint de s'attaquer au patrimoine royal, à coup d'appropriations et de privatisations de biens et droits extorqués tant à Pierre IV qu'à l'infant Jean ${ }^{9}$. Ce réquisitoire leur reproche enfin d'être une source récurrente d'injustices et d'avilissement de l'autorité royale puisqu'ils multiplieraient saisies et chantages arbitraires aux dépens des particuliers et détourneraient la justice retenue du roi à leur propre profit ${ }^{10}$.

En janvier 1384, sept mois après ces premiers échanges houleux et devant cette même assemblée, le roi d'Aragon continuait à défendre ses hommes, en affirmant qu'ils le conseillaient bien ${ }^{11}$; de leur côté, ses sujets lui rétorquaient vertement que l'état des affaires royales, du patrimoine et de la chose publique prouvait le contraire ${ }^{12}$. Ils attribuaient une fois de plus les mauvaises décisions royales aux mauvais conseillers qui continuaient, selon eux, à siéger aux conseils du roi et de son fils aîné, à s'entretenir avec eux en secret et, plus grave encore, leurs auraient dicté l'attitude à tenir ${ }^{13}$.

7 « en tractar e fer la pau derra ab Jenoveses posats ladonchs en gran necessitat sens alcuna necessitat de vos, senyor, e sens tot vostre profit no sens gran minua e deshonor vostra ».

8 « ab tot allo vostre patrimoni es diminuit en tant que es quasi a extrem de periclitacion. "

9 « fer se donar per lo dit senyor duc e per vos senyor Castells, viles, lochs, juridiccions, rendes e altres bens de vostre patrimoni »; " cascuns dels dits consellers e curials de vos senyor e de vostre primogenit se son molt enriquehits».

10 " en demanar, pendre e haver de les gens moltes e grans quantitats de diners e de joyes per via de corrupcions e subornacions »; " pervertir les sentencies e los juhis e affers de la vostra sacra audiencia e cort reyal $»$.

11 Dans l'article 3 de la cédule royale lue devant l'assemblée le 9 janvier 1384, le roi affirmait que « no ha loch lo protest fet contra sos consellers qui en res de les dites coses no han colpa com en aquelles e en totes altre hajen consellat et consellen lo dit senyor justament e be » (p. 235).

12 « si axi fos, mils fora o seria de vostres afers et de vostre patrimoni et de la cosa publica de vostres regnes et terres, et los vostres pobles no haurien rao de clamarse et de parlar de vostres consellers et officials » : cédule des sujets, lue au roi le 15 janvier 1384, en réponse à sa cédule du 9 (p. 238).

13 « Et per ço et en altra manera, appar que la dessus dita recomendacio dels dits consellers sia mils ordinacio o escriptura d'alcuns d'ells que paraules de vos, senyor, salva la dita reverencia car no es semblant que la alta saviesa vostra, que sobre aço ha a fer juhi et justicia dels dits consellers, dixes de certa sciencia, sens discussio d'aquella, tals paraules, les quals donen raonablement a la cort causa de dubtar en la dita faedora justicia, majorment com sia cert et notori que alcuns dels sospeses son de fet restituits en lurs officis de casa vostra et de vostre primogenit, et tots aquells, o la major part, 
Autant dire que faute de pouvoir incriminer directement le roi et son fils, le jugement des sujets à l'encontre des conseillers, accusés de graves abus d'autorité, était particulièrement sévère et intransigeant. On ne connaît pas encore la liste nominative complète des hommes ici visés, ni l'ampleur des fautes que l'on peut leur attribuer ${ }^{14}$; mais on peut souligner que l'historiographie s'accorde à considérer qu'ils jouent un rôle central dans la déroute de l'autorité royale aragonaise à la fin du XIV ${ }^{\mathrm{e}}$ siècle ${ }^{15}$. Ces violentes

et encara los versemblants sospenedors, son admeses per vos senyor, et vostre primogenit en vostres et seus consells o parlaments secrets » (p. 238).

14 Le 27 août 1383, Pierre IV consent à publier une liste d'officiers et serviteurs incriminés et suspendus (SESMA MUÑOZ, J. Á. (éd.), Acta curiarum Aragonum, n.33, pp. 216-217 et SANS I TRAVÉ, J. M., Cort General de Montsó, pp. 122-124, n.6) : pour les conseillers royaux, il s'agit des chambellans Ramón de Vilanova et Hugo de Santapau, des majordomes Pedro Jordán de Urriés et Ramón de Peguera, des promovedors Manuel d'Entença, Ramón de Cervera, Narcís de San Dionís et du prothonotaire Bernat de Bonastre; les conseilles du duc et de la duchesse de Gérone sont Pere Boyl, Francesc et Constança de Perrellós, Pere Planella, Johan Janer, Simón Sarçet, Bernat et Johan dez Pont, Bartomeu de Llunes, le trésorier ducal et Arnau de Perobonet ; certains d'entre eux sont brièvement cités dans NAVARRO ESPINACH G., « Consejeros influyentes y personas de confianza en el entorno cortesano de los reyes de Aragón (siglos XIII-XV) », dans Sesma Muñoz, J. Á. (coord.) La Corona de Aragón en el centro de su Historia. 1208-1458. La monarquía aragonesa y los reinos de la Corona, Saragosse, 2010, p. 149 ; une étude prosopographique poussée de l'entourage du roi et de son fils permettrait de connaître précisément le rôle et les responsabilités de chacun. Grâce à quelques études, on connaît néanmoins les contours généraux des partis en conflit, tant dans l'entourage du roi, de la reine Sibil-la de Fortià, que de l'infant Jean et de son épouse Violante de Bar: BOSCOLO, A., Sibilla di Fortià regina d'Aragona, Padoue, 1970, pp. 55-75 ; TASIS I MARCA, R., Pere el Ceremoniós i els seus fills, Barcelone, 1980, p. 105 ; TASIS I MARCA, R., Joan I. El rei caçador i músic, Barcelone, 1959, p. 108 ; VIERA, D., " Francesc Eiximenis's Dissension with the Royal House of Aragon », Journal of Medieval History, 22/3 (1996), pp. 252-255 et SESMA MUÑOZ, J. Á., « La nobleza bajomedieval y la formación del Estado moderno en la Corona de Aragón », dans La nobleza peninsular en la Edad Media, VI Congreso de Estudios Medievales, Ávila, 1999, pp. 345-430. Sur les dénonciations à l'encontre de Bartomeu Llunes, trésorier de l'infant Jean, voir BEAUCHAMP, A., « Les comptes des dispensers de l'infant Jean d'Aragon (1351-1386) et leur contrôle par le maestre racional : des discours interposés », à paraître dans Santamaria-Lemonde, A., Ce que compter veut dire. Le discours comptable du XIII au XV siècle (principautés, monarchies et villes occidentales). Les travaux de M. T. FERRER I MALLOL, sur la procédure de récupération des droits de juridiction cédés par les prédécesseurs de Martin $\mathrm{I}^{\text {er }}$ au profit de proches conseillers, donnent un aperçu de l'importance du phénomène d'aliénation du patrimoine royal : «El patrimoni reial i la recuperació dels senyorius jurisdiccionals en els Estats catalano-aragonesos a la fi del segle XIV », Anuario de Estudios Medievales, 7 (1970-1971), pp. 351-494; et « Les recopilacions documentals dels arxivers del rei per la recuperació del patrimoni real », dans Barrio Barrio, J. A. (éd.), Los cimientos del estado en la Edad Media. Cancillerías, notariado y privilegios reales en la construcción del Estado en la Edad Media, Alcoi, 2004, pp. 13-37.

15 Voir par exemple FERRER I MALLOL, M. T., « El patrimoni reial », pp. 351-359; 
dénonciations constituent en outre un des temps forts de ce que José Ángel Sesma Muñoz qualifie de « lutte pour le contrôle de l'appareil administratif et judiciaire du monarque », qui anime la fin du règne de Pierre IV et celui de ses deux successeurs, les rois Jean ${ }^{\text {er }}$ et Martin l'Humain ${ }^{16}$. Ce sont en tout cas des éléments tonitruants du bras de fer qui paralyse les débats des Cortes generales ouvertes à Monzón en juin 1383 jusqu'à leur clôture provisoire en juillet 1384 à Fraga, et le dialogue politique jusqu'à la mort de Pierre le Cérémonieux en $1387^{17}$.

En effet, tant qu'il ne s'attaque pas à « l'assainissement » de son entourage, les sujets réitèrent leurs accusations, surenchérissent même dans leurs demandes et refusent de lui offrir l'aide financière et militaire ainsi que les conseils qu'il requiert d'eux ; ils réclament de leur côté que le roi interdise aux officiers suspendus de le conseiller et d'approcher sa Maison et celle du duc, et qu'il poursuive les enquêtes nécessaires pour écarter d'autres mauvais serviteurs et conseillers; puis ils exigent de connaître le nom des enquêteurs. A son tour, Pierre IV refuse d'accéder à leurs demandes tant que les sujets ne satisferont pas les siennes ${ }^{18}$. Affirmant agir par devoir, pour préserver l'honneur du roi et de la Couronne ainsi que

ABADAL, R. d', Pere el Cerimoniós, p. 102 ; ou encore SABATÉ I CURULL, F., «El poder soberano en la Cataluña bajomedieval : definición y ruptura », dans Foronda, F., Genet, J. Ph, Nieto Soria, J. M. (dir.), Coups d'État à la fin du Moyen Âge ? Aux fondements du pouvoir politique en Europe occidentale, Madrid, 2005, pp. 498509 : reprenant une analyse traditionnelle des historiens de la Couronne d'Aragon il souligne que « en el último cuarto del siglo XIV, con el gobierno naufragando ante la incapacidad fiscal, las dificultades jurisdiccionales, las agresiones externas, las fracturas sociales y las crisis frumentarias, el Consejo Real es un avispero de conspiraciones, intrigas y corrupciones $»$ (p. 501). SESMA MUÑOZ, J. Á. , "Todos frente al rey », p. 83.

17 Sur ce " dialogue de sourds », voir en particulier HÉBERT, M., Parlementer. Assemblées représentatives et échange politique, pp. 69-72. Depuis le milieu du XIve siècle, les assemblées d'État sont, dans la Couronne d'Aragon, le lieu d'un dialogue politique de plus en plus conflictuel entre le roi et ses sujets. Leur contribution financière à la politique royale est au cœur des débats et y donne lieu à d'âpres négociations, comme le montre SÁNCHEZ MARTÍNEZ, M., " Negociación y fiscalidad en Cataluña a mediados del siglo XIV : las cortes de Barcelona de 1365 », dans Ferrer i Mallol M. T., Moeglin, J.-M., Péquignot, S., Sánchez Martínez, M. (éd.), Negociar en la Edad Media / Négocier au Moyen Âge, Barcelone, 2005, pp. 123-164. Voir aussi BEAUCHAMP, A., "L'administration des profertes du bras royal catalan ou les conditions de son soutien à Pierre le Cérémonieux (1350-1357) », dans Foronda, F. (dir.), Avant le contrat social. Le contrat politique dans l'Occident médiéval (XIII ${ }^{e}-X V^{e}$ siècle), Paris, 2011, pp. 481-505.

18 Ce processus de négociation, à coup de joutes oratoires et documentaires et à grand renfort d'arguments dilatoires, est étudié par HÉBERT, M., Parlementer. Assemblées représentatives et échange politique, pp. 377-453. 
la chose publique et la justice, les sujets entendent l'obliger à respecter leur alliance et reconnaissance mutuelle, en vertu desquelles il avait justement accepté de réunir les représentants de tous les états de la société de ses différents royaumes et territoires ${ }^{19}$.

Les dénonciations du mode de vie et surtout des mauvais conseils prodigués au roi par de mauvais conseillers et curiaux sont récurrentes dans les royaumes européens et dans la littérature politique à la fin du Moyen $\hat{A} g e^{20}$. Pour ne citer que trois brefs mais significatifs exemples aragonais, on peut rappeler qu'en 1289, Alphonse le Libéral lui-même considérait que la désagrégation du patrimoine royal était imputable à de mauvais conseillers ${ }^{21}$. Dès son accès au trône en 1336, les conseillers et officiers insoficientes de Pierre le Cérémonieux avaient fait l'objet de vives critiques d'un parti qui se voulait réformateur et dénonçait leur influence néfaste sur le gouvernement du jeune souverain ${ }^{22}$. Lesquels conseillers, et en particuliers aquells qui són de Rosselló, accusés de diverses extorsions et surtout de contrevenir sciemment au droit foral, furent directement incriminés par les Valenciens lors de la révolte de l'Union de 1347-1348 ${ }^{23}$; comme les Aragonais, ils demandaient

19 Le proceso de ces Cortes generales enregistre de multiples proclamations des motivations de l'assemblée qui, le 27 juin 1383, se dit par exemple animée par une «sana intencio e de bo e vertader zel a labor de nostre senyor Deu e honor e utilitat de vostra corona e a reparacio de vostre patrimoni e a bon estament de vostres regnes e terres e altres de la vostra cosa publica e a conservacio de justicia per la qual vos, senyor, regits et regnats ara e peravant en lochs anys ab exalçament de vostra reyal corona ", p. 205 ; le 15 janvier 1384, l'infant Martin rappelle encore que « la present cort [...] principalment es appellada et ajustada per be de justicia », p. 237.

20 LEMAIRE, J., Les visions de la vie de cour dans la littérature française de la fin du Moyen Âge, Paris-Bruxelles, 1994 ; AUTRAND, F, « De l'Enfer au Purgatoire : la Cour à travers quelques textes français du milieu du XIV à la fin du XVe siècle », dans Contamine, Ph. (éd.), L'État et les Aristocraties (France, Angleterre, Ecosse). XII ${ }^{e}-\mathrm{XVII}^{e}$ siècle, Actes de la table ronde organisée par le CNRS (Maison française d'Oxford, 26-27 septembre 1986), Paris, 1989, pp. 51-78 ; TELLIEZ, R., 'Per Potentiam officii'. Les officiers devant la justice dans le royaume de France au XIV siècle, Paris, 2005 (en particulier pp. 403-524) et WATTS J., The Making of Polities. Europe, 1300-1500, Cambridge, 2009, pp. 3-6, qui souligne combien les protestations et critiques à l'encontre des conseillers royaux et en défense de la chose publique s'expriment à de multiples reprises et sous des formes similaires, dans les royaumes européens des $\mathrm{XIV}^{e}$ et $\mathrm{XV}^{\mathrm{e}}$ siècles. Dans les domaines du roi d'Aragon, ce thème semble avoir été récemment étudié par GRIFOLL, I., "Veus crítiques contra el rei i la 'Vita Curialis' a la literatura catalana tardomedieval », communication (inédite à ce jour) prononcée lors du colloque international Authority and Resistance in England and the Crown of Aragon (1100-1750), Northumbria University, Newcastle, 28-29 novembre 2011.

21 Selon les mots de SABATÉ I CURULL, F, «El poder soberano », p. 500.

22 BEAUCHAMP, A., « Ordonnances et réformes de l'hôtel au début du règne de Pierre IV d'Aragon », Anuario de Estudios Medievales, $39 / 2$ (2009), pp. 566-569.

23 RODRIGO LIZONDO, M., " La Unión valenciana y sus protagonistas », Ligarzas, 7 
la destitution des conseillers du roi et revendiquaient le contrôle de leur nomination ${ }^{24}$.

Dans les années 1380, les accusations à l'encontre des conseillers du roi d'Aragon sont d'autant plus vives que les sujets ne s'en prennent pas à la corruption ou à la vita curialis en tant que telles ni ne se contentent de demander au roi de suspendre et d'interdire l'accès à sa maison à des mauvais éléments. Ils remettent directement en cause la place des conseillers aux côtés du roi et de son héritier désigné, ainsi que leur emprise sur les affaires royales. Au-delà de la question du choix de mauvais auxiliaires par le souverain, la critique des Corts generales, à ce stade du conflit, concerne le rôle des proches conseillers du roi d'Aragon dans le processus décisionnel et dans le gouvernement de la Couronne, quand la quasi-totalité des décisions émanent officiellement du seul souverain. À Monzón, ils dressent en somme le procès d'un gouvernement par conseil qui lui échapperait en partie, puisque malgré ses dires, ses conseillers auraient pris de grandes libertés et décidé euxmêmes du sort de la Couronne.

Pour mieux s'en prémunir, celui-ci avait pourtant soigneusement délimité le cadre et les modalités de la contribution des conseillers.

\section{L'INTERVENTION DES CONSEILLERS D'APRÈS LES ORDONNANCES DE PIERRE LE CÉRÉMONIEUX}

Les Ordinacions de la Casa i Cort, promulguées par le roi Pierre IV en 1344, définissent le profil, la charge et les responsabilités des serviteurs de la Maison et du gouvernement royal, au rang desquels les conseillers ${ }^{25}$. Elles déterminent qui peut être nommé conseiller et peut siéger au conseil royal, évoquent certaines obligations des conseillers et envisagent brièvement la tenue des séances du conseil ${ }^{26}$. Elles esquissent donc un portrait du rôle et des limites de leur action aux côtés du souverain. Multipliant les recommandations sur le bon comportement que les hommes du roi doivent adopter pour servir la

(1975), pp. 137-138 ; BAYDAL SALA, V., Els orígens de la revolta de la Unió al regne de València (1330-1348), Valence, 2013, pp. 267-268.

24 TASIS I MARCA, R., Les Unions de nobles i el Rey del Punyalet, Barcelone, 1960, p. 28 ; SIMÓN BALLESTEROS, S., «El acuerdo secreto firmado entre el rey Pedro IV y el noble aragonés Lope de Luna durante la segunda Unión (1347-1348) », Aragón en la Edad Media, 22 (2011), p. 253.

25 Ordinacions de la Casa i Cort de Pere el Ceremoniós, Gimeno, F., Gozalbo, D., Trenchs, J. (éd.), Valence, 2009. Lapport des Ordinacions à la connaissance du conseil est traité dans TATJER PRAT, M. T., " La potestad judicial del rey ».

Ordinacions de la Casa i Cort, chap. 88 et 89, pp. 174-175. 
chose publique et satisfaire la volonté divine, elle ne se réduisent cependant pas à une simple " compilation de 'job descriptions' bureaucratiques », ni seulement à un règlement sans référence au « comportement éthique du personnel de la cour $»^{27}$.

Le chapitre Dels consellers nostres précise ainsi qui peut porter le titre de conseiller. Parmi les officiers de l'Hôtel royal, le chancelier, le vicechancelier, les majordomes, les chambellans, le maestre racional, le trésorier, les promoteurs, les secrétaires sont considérés comme des « conseillers nés $»^{28}$; mais d'ajouter que portera ce titre toute autre personne que le roi jugerait digne ( encara altres quals que quals a aço conexerem merexedors ») ${ }^{29}$. Par cette simple formule, le souverain se ménage donc la possibilité d'ériger au rang de conseiller tout individu de son choix, quel que soit son profil ; mais à lui, et à lui seul, revient le choix de ceux qui l'aideront à prendre ses décisions. Aucune considération sur les choix politiques et stratégiques qui peuvent le pousser à choisir tel ou tel officier-« conseiller né » n'intervient bien sûr dans les ordonnances. Le chapitre titré De la manera del seer e proposar en consell nostre permet d'affiner cette dernière catégorie ${ }^{30}$. A l'aide d'une métaphore organique des plus traditionnelles, il décrit en effet la disposition spatiale que les conseillers doivent respecter lors des séances du conseil. Ils seront assis en cercle de part et d'autre du roi ; du fait de leur rôle majeur, les membres de la famille royale et les nobles, par ordre hiérarchique, siègeront à sa droite, tandis que les prélats et clercs s'assiéront eux à sa gauche $^{31}$. La liste des membres potentiels du conseil et de la companya de

27 Ces expressions sont de WITTLIN, C., « La sisena part del Dotzè de Francesc Eiximenis com a complement ètic a les Ordinacions de la Cort del Rei Pere el Cerimoniós », Butlletí de la Reial Acadèmia de Bones Lletres de Barcelona, 50 (2005-2006), p. 232. Ces aspects réglementaires et pratiques sont néanmoins bien présents : BEAUCHAMP, A., "Les Ordinacions de la Casa i Cort de Pierre IV d'Aragon et le nombre des serviteurs royaux », dans Beauchamp, A. (dir.), Les serviteurs du prince à la fin du Moyen Âge. Une approche quantitative, Madrid, 2013, pp. 43-56.

Le texte parle d'ailleurs « d'office de conseiller »: « a tan gran offici de consellers de la nostra serenitat real sien reebuts canceller e vicecanceller », Ordinacions de la Casa i Cort, p. 174.

29 Ordinacions de la Casa i Cort, p. 174.

30 Ordinacions de la Casa i Cort, pp. 174-175.

31 « axí com de l'humanal cors los membres de la part dreta del dit cors major nodriments reeben e per major força són reformats, axí los reyals, comtes, barons e altres cavallers de la part dreta de nostre cors en conseyl sien decorats ; e los prelats, e.ls altres clergues, de la part sinistra en lurs seyles sien hondrats ", Ordinacions de la Casa i Cort, p. 175. Le « cercle» du conseil - que les membres doivent quitter pour s'exprimer dans certaines conditions - est évoqué plus loin dans ce chapitre, qui n'est pas sans rappeler «l'ordonnancement des sièges et la hiérarchie des discours [...] arrangés par le roi lui-même » aux Cortes generales de 1383, dont témoigne le 
nostres conseylers ménage donc une place de choix aux princes de la Maison d'Aragon. Si ce chapitre - centré sur la façon dont les conseillers et personnes invités à assister au conseil peuvent y intervenir -, évoque aussi les nobles et clercs, il ne dit mot de la présence ni de la position, lors des séances, d'éventuels conseillers laïcs roturiers. Il ne spécifie pas non plus l'endroit où devront s'installer les « conseillers nés » qui pourraient ne pas être clercs ni nobles, tels le maestre racional, le trésorier et les secrétaires ${ }^{32}$. Une décennie plus tard, dans une ordonnance probablement émise depuis la Sardaigne au premier semestre 1355, Pierre IV évoque leur cas $^{33}$. Dans ce texte, qui veille notamment à préserver l'honneur des officiers royaux en ordonnant de les faire adouber avant leur nomination, le roi ordonne qu'aucun laïc ne soit reçu au conseil, s'il n'obtient pas le statut de rich hom ou de chevalier ou s'il n'est pas ciutadà honrat, trésorier, maestre racional, scrivà de ració ou prothonotaire ; il prévoit aussi que les nobles chevaliers auront la primeur, sur ceux qui n'auront pas été adoubés, par le siège qu'ils occupent et dans l'ordre de parole ${ }^{34}$. Le titre de conseiller, librement attribué par le roi, est donc ainsi réservé à une élite, elle-même très hiérarchisée et susceptible de le conseiller aussi souvent qu'il le requiert.

Selon les ordonnances, ce titre implique en effet que les bénéficiaires doivent être totalement disponibles pour leur souverain et ne peuvent s'absenter de l'endroit où il réside sans son autorisation. Cette obligation de présence ne constitue en rien une spécificité liée au statut des conseillers puisque dans les Ordinacions, elle s'impose aussi aux officiers de l'hôtel royal dont les absences et les modalités de remplacement sont définies ${ }^{35}$. Elle suppose néanmoins que les conseillers, quel que soit leur statut personnel, sont susceptibles d'être sollicités à tout moment par le souverain, pour l'aider à prendre ses décisions, l'éclairer, le guider. Ainsi, suivant ces

proceso de l'assemblée analysé par HÉBERT, M., Parlementer. Assemblées représentatives et échange politique, pp. 58-61.

32 Leur statut personnel n'est pas précisé dans les chapitres qui leurs sont respectivement consacrés, contrairement par exemple aux majordomes ou aux copers qui doivent être de condition noble, aux algutzirs, aux cavallerices ou encore aux deux des quatre promovedors qui doivent être chevaliers ou aux sobrecochs qui ne peuvent être recrutés que parmi les écuyers de lignage de chevalier.

33 Archivo de la Corona de Aragón, Cancillería, reg. 1529, fol. 45r-46r ; publiée par BOFARULL I MASCARÓ, P. de (éd.), dans Colección de Documentos Inéditos del Archivo General de la Corona de Aragón, t. VI, Barcelone, 1850, pp. 69-71.

34 «Item que en lo consell del senyor rey no sia reebut null hom lech si rich hom o cavaller fet no es o honrat ciutada o tresorer, maestre racional, escrivà de ració 0 prothonotari tinent los seus segells; e ordena et vol quels richs homens qui no seran cavallers en lo consell del dit senyor en seti et en parlar sien derrer dels cavallers ».

BEAUCHAMP, A., « Les Ordinacions de la Casa i Cort », pp. 49-52. 
ordonnances, les conseillers peuvent être amenés à fournir leur conseil au roi dans tout contexte, aussi bien en dehors que pendant les séances du conseil royal.

Celles-ci font plus particulièrement l'objet du chapitre consacré à l'étiquette et au protocole qui y régissent la prise de parole ${ }^{36}$. Il précise si les conseillers et autres intervenants au conseil doivent rester assis ou se lever pour s'exprimer, en fonction de leur rang et du titre auquel ils prennent la parole (en tant qu'avocat, au titre de leur office ou d'une commission du roi). De cette façon les ordonnances de 1344 veillent plus à préserver l'ordre et les préséances, à réguler l'influence des conseillers, qu'à définir les compétences du conseil ${ }^{37}$. Lordre de distribution de la parole au conseil offre à certains conseillers la possibilité de peser plus que les autres sur les décisions qui y sont prises. Or cette question fait l'objet de considérations complémentaires dans le chapitre consacré à la définition des tâches du chancelier. Il y apparaît comme le gardien des usages à faire respecter au conseil, qu'il peut présider en l'absence du roi ${ }^{38}$. En cas de débat au conseil, ledit chancelier doit veiller à faire s'exprimer les conseillers les plus importants (els majors) après les autres $^{39}$. Ce faisant, il permet d'une part à ces majors d'offrir un meilleur conseil en le formulant après avoir pris connaissance de celui émis par leurs collègues de moindre envergure ; mais il leur évite d'autre part d'être ridiculisés par ces derniers, qui s'exprimant en second lieu, pourraient corriger leurs propos. Cette hiérarchie de la parole est non seulement un moyen de ne pas heurter les susceptibilités, mais elle constitue aussi un gage

36 Chapitre titré De la manera del seer e proposar en consell nostre, évoqué plus haut (Ordinacions de la Casa i Cort, pp. 174-175).

37 Un conseiller agissant en tant qu'avocat devra par exemple se lever et sortir du cercle des conseillers pour parler; lorsqu'il agit à la demande du roi au titre de son office ou d'une commission spéciale, il peut en revanche demeurer assis pour s'exprimer (Ordinacions de la Casa i Cort, p. 175).

38 « volem que.l canceller ordon les altres coses les quals, present Nós en lo conseyl, seran faedores, manera de estar e de proposar e semblants coses, esguardants aquí estaments de persones segons la nostra ordinació sobre açò feta ", Ordinacions de la Casa i Cort, p. 121.

39 " que tota hora que en nostres conseyls se discutiran alscunes coses sobre las quals cascun dels conseylers clergues tan solament o cascun dels conseylers qualsque quals dir son conseyl convendrà, lo dit canciller injunga primerament als altres que no als majors que diguen ço que sabran conseylar, car meylor és que los dits dels altres sien corregits per los dits subsegüents dels majors que si los dits dels majors se corregien per los dits après subseguits dels altres; e si els mayors primerament dehïen aquelles coses que veurien ésser conseyladores, per aventura aytal occasion de mala absordidat se donaria que.ls altres, no volents per favor o no gosans per temor, partir d'aquelles lexarien a dir aquelles coses les quals cogitarien ésser conseyladores, l'enteniment propri en ornament subvertens », Ordinacions de la Casa i Cort, pp. 120-121. 
de qualité des conseils énoncés, d'autant que les conseillers moins primés s'exprimant en second pourraient ne pas oser dire leur pensée et laisser s'imposer de mauvais conseils. On lit donc dans ces ordonnances combien, au sein du conseil royal, la parole et les conseils émis par les conseillers ne peuvent être spontanés, ne peuvent librement s'imposer à l'oreille du roi et de l'assistance. On comprend en outre qu'au conseil comme en dehors, les conseillers ne doivent s'exprimer qu'après y avoir été invités par le souverain et aussi souvent qu'il le leur demandera.

On ne trouve d'ailleurs dans ces ordonnances de 1344 aucune définition de la fréquence des réunions du conseil. Elles ne font aucune allusion à l'ordonnance dite " de Huesca », promulguée par le roi Alphonse III d'Aragon en $1286^{40}$. Celle-ci indiquait que le conseil devait être convoqué chaque jour, mais que le roi n'était tenu d'y assister que les mardis et vendredis, jours de traitement des affaires les plus importantes. Cette mention affirmait implicitement qu'il s'en remettait en son absence à ses conseillers et leur déléguait les affaires courantes, sans néanmoins définir ce qui relevait de ces catégories. Ce silence du texte de 1344 laisse donc supposer que le roi entend que son conseil soit réuni aussi souvent que nécessaire, en sa présence ou non.

La quête d'ordre et de qualité, récurrente dans les Ordinacions, explique qu'elles s'intéressent aussi à la probité des conseillers. Parce que ces ordonnances ont vocation à définir ce qu'est le bon exercice des charges royales et des missions effectuées pour le roi, le chapitre Dels serveys encadre strictement les dons que les conseillers pourraient recevoir et qui seraient susceptibles d'influencer leur bon jugement, puisqu' "ils aveuglent les yeux des juges $»^{41}$. Afin d'éviter leur subornation, il leur est donc interdit de percevoir tous types de cadeaux, sous peine de perdre leur statut de conseiller et d'encourir une punition du roi. Sont exclus de cette interdiction les dons provenant de parents ou d'autres conseillers, ou reçus par le conseiller en

40 CARRERAS CANDI, F., "Redreç de la reial casa : ordenaments de Pere "lo gran" e Anfós "lo liberal" (segle XIII) », Boletín de la Real Academia de Buenas Letras de Barcelona, 5 (1909-1910), p. 105 ; voir aussi du même auteur, « Ordenanzas para la casa y corte de los reyes de Aragón », Cultura española, 2 (1906), pp. 327-338 ; TATJER PRAT M. T. , La Audiencia Real en la Corona de Aragón. Orígenes y primera etapa de su actuación, s. XIII y XIV, thèse soutenue à Barcelone en 1986 à l'Université de Barcelone sous la direction de J. M. Font i Rius, vol. 1, pp. 45-50 (éditée sous le même titre à Barcelone, en 2009).

${ }^{41}$ " volem e ordonam que.ls dits nostres conseylers, de dons e de serveys, e qui los huyls dels jutges ençeguen, si doncs no era de parent o de conseyler, se abstenguen ab acabament, cor oblació e molt pus fort recepció de don corch és de regiment ", Ordinacions de la Casa i Cort, p. 176. 
tant qu'ambassadeur du roi. De même, la dérogation vaut pour les dons de victuailles pour un montant maximal de 20 sous, voire de 50 sous annuels en fonction de la qualité du donateur ; mais pour les dons de bijoux le montant maximal admis est de 10 sous annuels. La peur de la corruption pousse en outre le souverain à refuser le moindre de ces avantages aux conseillers en charge de procès. Pour s'assurer de leur engagement à bien servir le roi, les Ordinacions leur imposent enfin de prêter serment de le conseiller au mieux, avec fidélité, probité et droiture, et de ne pas ébruiter les secrets partagés ${ }^{42}$.

On constate donc combien ce texte ne livre pas de définition de la place des conseillers et du conseil royal dans l'organigramme des pouvoirs qu'il esquisse par ailleurs. Il ne délimite pas non plus l'amplitude de leurs compétences, qui ne sont que très partiellement évoquées, puisqu'au sein ou hors du conseil, les conseillers peuvent être amenés à traiter toute affaire concernant le gouvernement de la Couronne et les intérêts du souverain. C'est-à-dire que bien que le gouvernement soit le premier enjeu du conseil, selon ces ordonnances, il n'y est pas institutionnalisét3. Le conseil du roi d'Aragon, comme celui de ses contemporains, demeure bien un " organe mouvant »dont le roi serait le seul maître, comme il affirme l'être de ses conseillers qu'il peut faire et défaire, solliciter à sa guise, pour l'aider à décider et à mieux gouverner ${ }^{44}$. A lire ce texte, l'intervention des conseillers et du conseil dans le processus décisionnel serait donc un élément hautement bénéfique pour le roi et pour son pouvoir, mais un élément étroitement dépendant de la volonté royale.

$$
* \quad * \quad *
$$

42 « ordenam que.ls nostres consellers a Nós per sagrament prometre sien tenguts que aytant com poran a Nós bon conseyl e feel daran, dients aquelles coses que sabran conseylladores, favor, odi e temor de tota persona de tot foragitats. Encara secret de nostre conseyl servaran », Ordinacions de la Casa i Cort, p. 174.

43 C'est pourquoi François Foronda doute d'une quelconque filiation entre le Consejo de Castilla, tel qu'il est institutionnalisé aux Cortes de Valladolid en 1385, et le conseil du roi d'Aragon, tel qu'il est décrit dans les ordonnances de 1344 (FORONDA, F., «El consejo de Jetró a Moisés (Ex.18, 13-27) o el relato fundacional de un gobierno compartido en la Castilla Trastámara », dans Boucheron P., Ruiz Gómez F. (coord.), Modelos culturales y normas sociales al final de la Edad Media. Actas del coloquio organizado por el LAMOP, EFR, Casa de Velázquez, Universidad de Castilla-La Mancha (Madrid-Almagro, 2004), Cuenca, 2009, p. 82, n.16). Sur l'organisation institutionnelle et humaine du Consejo de Castilla, voir DE DIOS, S., El Consejo Real de Castilla (1385-1522), Madrid, 1982.

44 L'expression est de KRYNEN, J., L'Empire du roi. Idées et croyances politiques en France, $X I I I^{e}-X V^{R}$ siècle, Paris, 1993, p. 221. 
Les ordonnances prétendent donc encadrer les possibilités d'intervention des conseillers dans les affaires royales. Choisis par le souverain, ils ne pourraient donc le conseiller et concourir au gouvernement de la Couronne qu'à sa demande et suivant sa volonté. Tout en demeurant assez vagues sur l'ampleur des responsabilités qui peuvent leur être confiés, elles en font des éléments fondamentaux du service du roi et, ce faisant, prescrivent un gouvernement par conseil mesuré et rassurant, conforme au modèle traditionnellement offert aux princes par la littérature politique $e^{45}$.

Pierre le Cérémonieux lui-même se l'est vu implicitement prescrire vers 1357-1358, dans le miroir des princes que lui dédie son oncle l'infant Pierre, comte de Ribagorza et des Montagnes de Prades. C'est ainsi que selon son De vita, moribus et regimine principum, pour faire régner un gouvernement tempéré, le bon roi ne doit pas manquer de solliciter la sagesse de ses conseillers, à l'avis desquels il doit savoir se rendre, puisque " in hoc enim magnam ostendit princeps humilitatem, qui cum sit in consilio summus humiliet se subdens consilio ${ }^{46}$. Lesquels conseillers doivent briller par leur probité et leur désintéressement personnel, pour mieux servir le prince et la chose publique $^{47}$. Cette norme est omniprésente dans le monumental Dotzè llibre del Crestià (vers 1385-1392) du franciscain Francesc Eiximenis qui ne consacre pas moins de 25 chapitres de sa sixième partie au conseil et aux conseillers royaux ${ }^{48}$. Or Curt Wittlin a montré de façon très convaincante que loin d'être une cuvre théorique, détachée de toute actualité, le Dotzè

45 KRYNEN, J., L'empire du roi, p. 220-223.

46 De vita, moribus et regimine principum, cap. VII « De principe quod habeat rectum iuditium », dans BEAUCHAMP, A. (éd.), Le De vita, moribus et regimine principum, miroir des princes rédigé par l'infant Pierre d'Aragon (v.1357-1358) (BN Madrid, mss $n^{\circ} 12987$ ), édition en ligne sur le site Narpan.net : Espai de Literatura i Cultura Medieval : http://www.narpan.net/ben/indexderegimine.htm (Consulté le : 20/02/2014) ; BEAUCHAMP, A., " De l'action à l'écriture : le De regimine principum de l'infant Pierre d'Aragon (v. 1357-1358) », Anuario de Estudios Medievales, 35/1 (2005), pp. 233-270.

47 De vita, moribus et regimine principum, cap. III, « De conditione et regimine consiliariorum et aliorum officialium principis ».

48 Parmi de nombreux passages du Dotzè del Crestià invitant tout bon roi à solliciter et à suivre l'avis de ses conseillers, on peut citer celui-là : « « si.t vols ben regir en tots tos fets, ans de totes tes obres vaja davant consell ferm. Et diu a propòsit ferm, car consell que gran senyor aja difinit e determenat de fet se deu seguir e, si no u fa, ell fa gran legea en sa magestat e gran minva a sos consellers, e ensenya que és de mal recapte e de poch de bé » (Francesc Eiximenis, Dotzè llibre del Crestià (Segona part), Wittlin C. et alii (éd.), Gérone, 1987, cap. DCCXXIII « Com se deu avisar lo príncep en les coses grans, ne quals coses són dites ésser grans », p. 110); sur la définition du profil des conseillers sur lesquels le roi doit s'appuyer selon Eiximenis, voir WITTLIN, C., " La sisena part del Dotzè », pp. 239-242 et n.20. 
ne cesse de multiplier les allusions aux princes aragonais de son temps. De façon dissimulée, Pierre IV et Jean I ${ }^{\mathrm{er}}$, et leurs déboires des années 13831388 sont particulièrement visés pour mieux les critiquer, en particulier en ce qui concerne leurs choix politiques et les abus de leurs conseillers et serviteurs ${ }^{49}$.

Les dénonciations des Cortes generales de Monzón et la question de l'action néfaste des mauvais conseillers continuent en effet d'empoisonner les relations entre les sujets et la royauté sous le règne des successeurs de Pierre le Cérémonieux. Ajournées en juillet 1384, une fois l'engagement pris par ce dernier de poursuivre les officiers et conseillers suspendus et d'enquêter plus avant sur les méfaits de ses serviteurs ${ }^{50}$, ces Cortes sont rouvertes en novembre 1388, sous la présidence de Jean $\mathrm{I}^{\mathrm{er}}$. Mais le nouveau roi d'Aragon doit désormais faire face à une surenchère de la part des sujets qui souhaitent mettre sous tutelle le gouvernement royal en désignant euxmêmes une partie des conseillers royaux et des membres de l'Hôtel ${ }^{51}$. Ces mêmes conseillers et serviteurs, toujours jugés responsables de la déroute de la royauté, font l'objet d'un retentissant procès à la mort de Jean $\mathrm{I}^{\mathrm{er52}}$.

49 WitTlin, C., «El rei Pirro de Roma en el "Dotzè de Cristià" de Francesc Eiximenis. Crítica encoberta de la política sarda del rei Pere de Catalunya », Anuario de Estudios Medievales, 25/2 (1995), pp. 647-657 et «Eiximenis i la destitució dels reis Pirro, Trocus de Pèrsia, Torpeius, Salopi i Lleó : critiques encobertes del rei Pere en el Dotzè », dans Bover i Font, A., Lloret, M. R., Vidal-Tibbits, M. (éd.), Actes del novè Col-loqui d'Estudis Catalans a Nord-Amèrica: (selected proceedings) (Barcelona 1998), Montserrat, 2001, pp. 509-527 ; la question des abus des conseillers royaux fait aussi l'objet de nombreux passages de la Doctrina compendiosa que C. WITTLIN attribue désormais au frère Ramon Soler plutôt qu'à Eiximenis, comme de certains chapitres de son Pastorale, initialement dédié au cardinal Jaume d'Aragon : "'Quae maxime damnant animas principum' : Funf antimonarchische Kapitael im Pastorale des Francesc Eiximenis », Zeitschrift fiir Katalanistik, 2 (1989), pp. 102-103 ; D. VIERA souligne combine cette critique est aussi présente dans le Llibre de les dones du franciscain : "Francesc Eiximenis's Dissension with the Royal House of Aragon ", Journal of Medieval History, 22/3 (1996.), p. 251.

50 SESMA MUÑOZ, J. Á. (éd.), Acta curiarum Aragonum, pp. 262-263.

51 SESMA MUÑOZ, J. Á. , "Todos frente al rey », pp. 84-94. Cette audacieuse solution est aussi évoquée par Francesc Eiximenis, selon WITTLIN, C., «Francesc Eiximenis i el secret nacional de les Ordinacions de la Cort del rei Pere el Cerimoniós », Butlletí de la Reial Acadèmia de Bones Lletres de Barcelona, 51 (2007-2008), pp. 81-82.

52 MITJÀ, M., " Procés contra els consellers domèstics i Curials de Joan I, entre ells Bernat Metge », Boletín de la Real Academia de Buenas Letras de Barcelona, 27 (19571958), pp. 375-417 ; KAGAY, D., « Poetry in the dock: the court culture of Joan I on trial (1396-1398) », dans Kagay, D., War, Government and Society in the Medieval Crown of Aragon, Aldershot, 2005, pp. 48-99 ; FERRER I MALLOL, M. T., « Lluites de bandols a Barcelona en temps del rei Martí l'Humà », Estudis d'historia medieval, 1 (1969), pp. 77-94, et SABATÉ I CURULL, F., « El poder soberano » envisagent plus particulièrement la question des luttes de partis. 
C'est dire si malgré les prescriptions des ordonnances, le rôle des conseillers ne devait pas se limiter à conseiller le roi, qui s'en remettait largement à eux pour gouverner, sans toujours viser toutes les étapes et mesurer les conséquences de leurs actions. C'est ce que devra montrer une étude circonstanciée de la place des conseillers dans le gouvernement de la Couronne d'Aragon, ainsi que de leur rôle individuel. 